\title{
Communicating beyond the conventional functions: an assessment of newspaper announcements
}

\begin{abstract}
By default, all announcements notify listeners or readers of a given event, be it social, cultural, or religious. Newspaper personal or non-product advertisements tend to make audiences focus on its sponsors or the subject-matter of the announcements. Most personal announcements fall within the homely discourse genre. Scholars believe that careful examination of these types of announcements reveals further communications achieved beyond its primary functions and literal wordings. These communications could be within sociocultural or religious contexts. This critique reviews scholarly literature on how language is employed in a variety of newspaper announcements to convey much deeper sociocultural, religious, or political messages. The review focuses two types of genres, viz: congratulatory, and obituary and death announcements. Findings reveal that in congratulation and thank-you messages, a higher level of communication is achieved through various segments of such announcements, i.e. the use of multimodal, its position in the newspaper, etc. It shows how the announcements serve certain communicative purposes and how they are being influenced by factors such as gender and socioeconomic class. On the other hand, the literature reviewed on obituary and death announcements discloses how language is being utilized in the advent of death to communicate, not only to the deceased, but with wider audiences. The appraisal demonstrates how pages of newspaper death notice become platforms for eradicating social divisions and fighting the privileged groups. Another part of the evaluation discusses some contrasting conflicting views on the question of invoking God and religion in death rituals. As deceased persons are being celebrated and seen as martyrs in given cultures, others subscribe to notions that perceive death as personal choice and mere departure from the world. It is therefore recommended that more empirical works be conducted on congratulatory and other happy ceremony category of the newspaper announcements as the literature reviewed, so far, shows a very few scholarly works in that aspect of the genre.
\end{abstract}

Keyword: Newspaper announcements; Genre; Homely discourse; Congratulation; Obituary; Communicative function 